\title{
Psychometric Properties of the Coping Inventory for Stressful Situations in Korean Adults
}

\author{
Yoonmi Choi ${ }^{1}$, Eunsoo Moon ${ }^{1,2} \bowtie$, Je Min Park ${ }^{1,2}$, Byung Dae Lee ${ }^{1,2}$, \\ Young Min Lee ${ }^{1,2}$, Hee Jeong Jeong ${ }^{1}$, and Young In Chung ${ }^{3}$ \\ 1'Department of Psychiatry, Medical Research Institute, Pusan National University Hospital, Busan, Republic of Korea \\ ${ }^{2}$ Department of Psychiatry, Pusan National University School of Medicine, Yangsan, Republic of Korea \\ ${ }^{3}$ Department of Psychiatry, Pusan National University Yangsan Hospital, Yangsan, Republic of Korea
}

Objective The Coping Inventory for Stressful Situations (CISS) is a globally recognized measure of stress coping methods. However, research into the applicability of the CISS in a Korean context is still in its infancy. The aim of this study is to assess and report the validity of the CISS in Korean adults for the first time.

Methods Three hundred and two Korean adults who currently have no distressing problems requiring psychiatric treatment completed the Korean version of the CISS. Principal component analysis was used to extract factors in the process of exploratory factor analysis.

Results The result displayed a clear pattern matrix, and a high level of internal consistency was shown by Chronbach's alpha. The items classified under task-oriented and emotion-oriented coping presented adequate factorial validity, and only three items grouped under avoidance-oriented coping loaded poorly or loaded onto factors differing from the original.

Conclusion These results seem to indicate that the CISS may indeed be both applicable and useful in gauging the coping styles of Korean adults. However, the ambiguous meanings of certain items under avoidance-oriented coping would require adjustment for the purposes of future study.

Psychiatry Investig 2017;14(4):427-433

Key Words CISS, Coping, Factor analysis, Psychometrics, Validation.

\section{INTRODUCTION}

It has been noted that, when a person confronts stressful situations in life, that individual's coping strategy plays a significant role in their ability to adequately adapt to the situation. ${ }^{1}$ It is also true that the same stressors do not cause the same-if any-dysfunction in every individual, but rather the occurrence of dysfunction depends on each individual's coping responses and social resources as Billings et al. ${ }^{2}$ stated. Campbell-Sills et al. ${ }^{3}$ noted people who are neurotic and highly sensitive to stressors, and who tend to use emotion-orient-

Received: April 19, 2016 Revised: July 8, 2016

Accepted: August 24, 2016 Available online: May 17, 2017

$\triangle$ Correspondence: Eunsoo Moon, MD

Department of Psychiatry, Pusan National University Hospital, Pusan National University School of Medicine, 179 Gudeok-ro, Seo-gu, Busan 49241, Republic of Korea

Tel: +82-51-240-7303, Fax: +82-51-248-3648, E-mail: esmun@hanmail.net

(a) This is an Open Access article distributed under the terms of the Creative Commons Attribution Non-Commercial License (http://creativecommons.org/licenses/by$\mathrm{nc} / 4.0$ ) which permits unrestricted non-commercial use, distribution, and reproduction in any medium, provided the original work is properly cited. ed rather than task-oriented coping, are more vulnerable and have lower overall resilience. Further, it has been documented that patients with mood disorders tend to use emotionoriented coping strategies, instead of other alternatives such as problem-solving approaches or social interaction, which are preferred by people regarded as healthy. ${ }^{4}$ Some emotional stress responses such as rumination are found to be related to a vicious cycle of self-absorption and the experience of an increased degree of hopelessness. ${ }^{5}$ Additionally, it seems that the absence of adequate coping skills entails the increased likelihood of both mental disorders and suicidal behavior. ${ }^{6}$ For these reasons, we hold that enhancing the coping skills of patients is just as important as medication when it comes to helping them adapt to their circumstances in the long-term. This is particularly important when one considers that the inadequacy of the coping strategies of any given individual-be they psychiatric in-patient, former patient in remission, or member of the general public-increases the likelihood that that individual will experience either the worsening of their current condition, or the onset of problems as the result of psy- 
chological maladaptation. ${ }^{7-10}$ In addition, certain studies of the general population support the view that those who exhibit symptoms of depression, along with those at high risk of mental disorder, tend to use predominantly emotion- and avoidance-oriented coping strategies, as opposed to those which are more task-oriented or problem-solving in nature. ${ }^{11,12}$

To help clinical patients improve their coping skills overall, we must first identify their preferred coping strategy or strategies. The Coping Inventory for Stressful Situations (CISS) has proven itself to be a psychometrically valid and reliable self-reporting instrument when it comes to the assessment of coping skills. It was originally developed by Endler and Parker ${ }^{13}$ in North America, and has since been widely used not only in Western countries, but globally-including in such countries as Japan and Malaysia which fact is, it shall be seen, highly relevant to our study. The instrument itself consists of 48 items and assesses a variety of coping styles which categorize the types of behavior to be measured, as is indicated by the name of its prototype-the 'Multidimensional Coping Inventory (MCI)'. These types were originally designated as 'task-oriented,' 'emotion-oriented', and 'avoidance-oriented' coping. In addition, the avoidance-oriented coping type itself contains two subscales, these being 'distraction' and 'social diversion'. In terms of reliability, six-week test-retest reliabilities were found to be adequate in the study by Endler and Parker, ${ }^{14}$ as their correlations ranged from 0.51 to 0.73 , and the Cronbach's alpha values ranged from the high 80 s to low 90 s.

Numerous studies have been conducted internationally using the CISS, and yet data concerning its applicability within the Republic of Korea remain scarce. However, due to many such studies conducted over the last 25 years, suggestions for the improvement of the instrument itself have been advanced, including new factor models (e.g., 4-6 factor models), and a shorter version containing only 21 items. ${ }^{15-17}$ A number of studies focusing on cross-cultural conditions have also been conducted using localized versions of the CISS, the majority of the results of which have been found to be psychometrically valuable. ${ }^{18-21}$

In Korea, a validation study of the CISS in high-school students has been conducted using exploratory factor analysis by Park et al. ${ }^{22}$ It seems that the three underlying factors ascertained by this study replicated the established structure of the CISS in previous studies in North America, and internal consistency reliabilities were also sufficient. However, 9 of the 48 items produced unclear results due to cross-loadings, and independence among the three factors was not seen in adolescent males. Another validation study of the CISS in AfricanAmerican adolescents was also reported in Korea in the same year, ${ }^{23}$ and this study also showed the coefficients of Cronbach's alpha to be sufficient. However, many items proved lim- ited when used in non-white adolescents.

The CISS was originally administered to adults in North America, ${ }^{14,24}$ but no validation study on the CISS in Korean adults has been conducted as yet. Even though the applicability of the CISS to adolescents in a cross-cultural setting may not have proved satisfactory in the studies mentioned above, we felt that a separate study should be conducted in order to ascertain the level of its applicability to Korean adults-all the more so when one considers the indications that the CISS instrument ought to maintain its applicability in an international setting. ${ }^{25}$ and that it has indeed proved applicable in settings, including Japan, which could be said to have more in common with Korea than North America when it comes to cultural setting and societal structure. ${ }^{11,18,20}$ While we consider it uncontroversial to say that the CISS has so far proved helpful in assessing a given patient's individual style of coping with stressful situations, no reference value for Korean adults has yet been established-and it would be problematic, to say the least, to draw comparisons between the results of middle aged or elderly people and those of high school students. Hence, a study for acquiring a reference range for Korean adults is required. And so, with these considerations in mind, the main focus of this study is to report the validity of the CISS in its application to Korean adults for the first time.

\section{METHODS}

\section{Participants}

Participants were recruited from among a random sample of people encountered by the research team in public places, such as: department stores, transit stations, hospitals, places of business, and educational institutions, between January 2012 and January 2013. The purpose and procedures of the study were explained to each participant prior to commencement, and written informed consent was obtained from each individual concerned. The criteria for inclusion were: 1) Participants must be $18^{-} 65$ years old (mean 33.2, $\mathrm{SD} \pm 9.3$ ) and 2) Participants must show no distressing problems requiring psychiatric treatment. The exclusion criteria were specified as follows: 1) Anyone previously admitted to a psychiatric ward of any description; 2) Persons who have serious medical conditions; 3) Individuals with organic mental disorders and/or mental retardation, and 4) Individuals unable to read and/or write. In order to ascertain the eligibility of subjects for participation, questions regarding the inclusion and exclusion criteria were asked upon first contact with prospective participants, again upon obtaining informed consent, and demographic information was also taken via a form attached to the questionnaire itself, in order to make doubly sure that no exclusion criteria had been breached. A total of 302 Korean adults (149 
men and 153 women) participated in the study. The questionnaire was carried out once individually. The study protocol was approved by the Pusan National University Hospital Institutional Review Board.

\section{Instrument}

The CISS is a 48 -item self-report inventory that measures stress coping strategies in three dimensions-namely task-, emotion-, and avoidance-oriented coping, each dimension itself comprising 16 items. Task-oriented coping entails treating a stressful situation as a problem to be solved, and tackling it directly as such; emotion-oriented coping aims at mitigating emotional stress, and avoidance-oriented coping centers on the postponement of dealing with current problems. In this study, we used the same version of the CISS as was used in the 2000 study of Park et al..$^{22}$ also in the form of a pencil-andpaper questionnaire, but this time adapted for use with Korean adults.

\section{Statistics}

Exploratory factor analysis was used to determine the underlying factor structure of the CISS. Principal component analysis was used to extract factors. The number of factors was determined by examination of the scree plot and size of the eigenvalues. A promax rotation was then performed to create a more readily interpretable factor structure, and 0.32 was chosen as the minimum loading of an item for interpretation. Cronbach's alpha was used to examine the internal consistency reliability. These statistical analyses were performed using the Statistical Package for Social Sciences, version 18.0 (SPSS Inc., Chicago, IL, USA). Congruence coefficients were computed using the following formula which was presented in the book by Jensen ${ }^{26}: r_{c}=\frac{\sum X Y}{\sqrt{\sum X^{2} \sum Y^{2}}}$

\section{RESULTS}

The scree plot presented 11 factors with eigenvalues greater than 1.0. Only the first three factors were finally selected for the principal component analysis of all 48 items, as the elbow of the scree plot clearly indicated that these three factors account for most of the variance.

The total sample means, standard deviations and Cronbach's alpha values for the three factors of the CISS are shown in Table 1.

Table 2 presents the correlation coefficients between each factor. As the factor correlation matrix displayed a correlation of above 0.32 between the 'task' and 'avoidance' factors, oblique rotation was applied as Tabachnick and Fidell suggested. ${ }^{27}$ Since the CISS measures a person's behavior, and
Table 1. Descriptive statistics and the internal consistency reliabilities of each main factor of the CISS

\begin{tabular}{lcc}
\hline \multicolumn{1}{c}{ CISS scales } & Total mean $(\mathrm{SD})$ & Cronbach's $\alpha$ \\
\hline Task & $50.54( \pm 10.60)$ & 0.92 \\
Emotion & $34.89( \pm 9.36)$ & 0.88 \\
Avoidance & $42.09( \pm 10.21)$ & 0.86 \\
Total & $127.52( \pm 22.27)$ & \\
\hline
\end{tabular}

CISS: Coping Inventory for Stressful Situations, Task: task-oriented coping factor, Emotion: emotion-oriented coping factor, Avoidance: avoidance-oriented coping factor, Total: total scores of the CISS

Table 2. Component (factor) Correlation Matrix of the CISS

\begin{tabular}{lccc}
\hline Component (factor) & 1 (task) & 2 (emotion) & 3 (avoidance) \\
\hline 1 (task) & 1.000 & & \\
2 (emotion) & 0.148 & 1.000 & \\
3 (avoidance) & 0.340 & 0.269 & 1.000 \\
\hline
\end{tabular}

Extraction Method: Principal Component Analysis. Rotation Method: Promax with Kaiser Normalization was applied as a correlation $\geq 0.32$ between two factors was seen

task- and avoidance-oriented coping strategies are inherently behavioral in nature-as are some forms of emotion-oriented coping-it is natural to expect that correlations between the factors should arise. As has been pointed out before, Costello et al. ${ }^{28}$ also stated that such cross-factorial correlation is to be expected to a greater or lesser extent due to the fact that human behavior is not easily compartmentalized into perfectly distinct categories.

The final factor matrix, eigenvalues, and explained variances are indicated in Table 3 . The matrix yielded the clearest results when subjected to principal component analysis with promax rotation. To determine whether or not a four-factor model would provide clearer results, this method of analysis was also used. However, it showed more cross-loaded items and misplacements than the three-factor method.

All 16 items of factor 1 (i.e., task-oriented coping; the 'task' factor) showed higher factor loadings (0.50 and over) and clearly loaded compared to the items of two other factors. There were no items that cross-loaded on factor 1 , but item 35 ('Talk to someone whose advice I value'), which was supposed to belong to factor 3 (that of avoidance-oriented coping) on the basis of Endler and Parker's earlier studies, was in fact more strongly related to task-oriented coping than to its original factor. Similarly, all 16 items of factor 2 (emotionoriented coping) also showed sufficient factor loadings. Only item 34 ('Focus on my general inadequacies') cross-loaded on factor 1 , but this nevertheless seems acceptable, since its factor 1 loading score was not of any great significance, and was in fact smaller than the score recorded for factor 2 . As for the factor 3 (the 'avoidance' factor), the factorial validity 
Table 3. The final matrix

\begin{tabular}{|c|c|c|c|c|}
\hline & \multirow{2}{*}{ CISS item } & \multicolumn{3}{|c|}{ Factors } \\
\hline & & 1 (task) & 2 (emotion) & 3 (avoidance) \\
\hline $\mathrm{T}$ & 43. Come up with several different solutions & 0.844 & & \\
\hline $\mathrm{T}$ & 41. Get control of the situation & 0.782 & & \\
\hline $\mathrm{T}$ & 10. Outline my priorities & 0.740 & & \\
\hline $\mathrm{T}$ & 02. Focus on the problem and see how I can solve it & 0.725 & & \\
\hline $\mathrm{T}$ & 27. Think about the event and learn from my mistakes & 0.718 & & \\
\hline $\mathrm{T}$ & 42. Make an extra effort to get things done & 0.712 & & \\
\hline $\mathrm{T}$ & 21. Determine a course of action and follow it & 0.696 & & \\
\hline $\mathrm{T}$ & 36. Analyze the problem before reacting & 0.680 & & \\
\hline $\mathrm{T}$ & 47. Try to be organized for the next time like this & 0.675 & & \\
\hline $\mathrm{T}$ & 39. Adjust my priorities & 0.644 & & \\
\hline $\mathrm{T}$ & 24. Work to understand the situation & 0.636 & & \\
\hline $\mathrm{T}$ & 46. Use the situation to prove my ability & 0.623 & & \\
\hline $\mathrm{T}$ & 26. Take corrective action immediately & 0.599 & & \\
\hline $\mathrm{T}$ & 06. Do what I think is best & 0.577 & & \\
\hline $\mathrm{T}$ & 15. Think about how I have solved similar problems & 0.542 & & \\
\hline $\mathrm{T}$ & 01. Schedule my time better & 0.525 & & \\
\hline A & 35. Talk to someone whose advice I value & 0.423 & & \\
\hline $\mathrm{E}$ & 14. Become very tense & & 0.805 & \\
\hline $\mathrm{E}$ & 08. Blame myself for having gotten into this situation & & 0.790 & \\
\hline $\mathrm{E}$ & 13. Feel anxious about not being able to cope & & 0.758 & \\
\hline $\mathrm{E}$ & 22. Blame myself for not knowing what to do & & 0.746 & \\
\hline $\mathrm{E}$ & 07. Preoccupied with aches and pain & & 0.733 & \\
\hline $\mathrm{E}$ & 19. Become very upset & & 0.654 & \\
\hline $\mathrm{E}$ & 17. Blame myself for being too emotional & & 0.652 & \\
\hline $\mathrm{E}$ & 30. Worry about what I should do & & 0.594 & \\
\hline $\mathrm{E}$ & 25. Freeze and don't know what to do & & 0.594 & \\
\hline $\mathrm{E}$ & 05. Blame myself for procrastinating & & 0.570 & \\
\hline $\mathrm{E}$ & 34. Focus on my general inadequacies & 0.387 & 0.470 & \\
\hline $\mathrm{E}$ & 38. Get angry & & 0.466 & \\
\hline $\mathrm{E}$ & 28. Wish that I could change what happened or... & & 0.458 & \\
\hline $\mathrm{E}$ & 45. Take it out on others & & 0.434 & \\
\hline $\mathrm{E}$ & 16. Tell myself "it's not really happening" & & 0.407 & \\
\hline $\mathrm{E}$ & 33. Tell myself "it will never happen again" & & 0.375 & \\
\hline A & 11. Try to sleep & & 0.334 & \\
\hline A & 44. Take time off and get away from the situation & & & \\
\hline A & 23. Go to a party & & & 0.851 \\
\hline A & 29. Visit a friend & & & 0.795 \\
\hline A & 20. Buy myself something & & & 0.742 \\
\hline A & 40. See a movie & & & 0.668 \\
\hline A & 09. Window shop & & & 0.657 \\
\hline A & 18. Go out for a snack or a meal & & & 0.640 \\
\hline A & 37. Phone a friend & & & 0.629 \\
\hline A & 31. Spend time with a special person & & & 0.621 \\
\hline
\end{tabular}




\begin{tabular}{|c|c|c|c|c|}
\hline & \multirow{2}{*}{ CISS item } & \multicolumn{3}{|c|}{ Factors } \\
\hline & & 1 (task) & 2 (emotion) & 3 (avoidance) \\
\hline A & 48. Watch TV & & & 0.552 \\
\hline A & 04 . Try to be with other people & & & 0.437 \\
\hline A & 32. Go for a walk & 0.344 & & 0.426 \\
\hline A & 12. Treat myself to a favorite food or snack & & & 0.408 \\
\hline \multirow[t]{3}{*}{ A } & 03. Think about the good times in the past & & & 0.356 \\
\hline & Eigenvalues & 10.226 & 6.101 & 3.751 \\
\hline & Explained variance & 21.304 & 12.711 & 7.814 \\
\hline
\end{tabular}

Only factor loadings greater than or equal to 0.32 are presented. Factor 1, 2, and 3 with large eigen values were identified, accounting for $21.304,12.711$, and $7.814 \%$ of the total variances, respectively. T: task-oriented coping, E: emotion-oriented coping, A: avoidance-oriented coping (This categorization is based on that of the English CISS established by Endler and Parker)

proved adequate in all but three cases: two items (35 and 11) loaded on factors other than the original, and item 44 did not show significant loading on any given factor.

In addition, separate factor analyses for male $(n=149)$ and female $(n=153)$ adults were also performed to examine the congruence coefficients. They were $0.972,0.960$, and 0.933 for factors 1,2 , and 3 respectively.

\section{DISCUSSION}

The results of this validatory study of the CISS showed general support of the earlier study conducted in North America. ${ }^{13,14}$ The congruence coefficients and internal consistency reliabilities of the three factors used were all high and the pattern matrix clearly showed three distinct factors; there were only three items that did not load on their original factors. Although these three items are unlikely to have much effect on the general results of the assessment, they may have insufficient face and factorial validity, as explained below.

The most significant anomaly was item 35 (i.e., 'Talk to someone whose advice I value'), which loaded on the task factor, but not its original factor (the avoidance factor). Regarding this problem, Cosway et al. ${ }^{29}$ have stated that, '[I]t is premature at this time to suggest that some items be rewritten.' However, the cross-loading of this item is to be found in several studies conducted to date. ${ }^{4,19,29}$ Given the number of studies, including our own which report that item 35 tends to load on the task factor, it seems justified to view this particular item as an attempt to confront and solve a problem (i.e., seeking out valuable advice), rather than simply avoid it. In support of this view, we would point out the significant distinction between 'Talking to someone whose advice I value' and merely 'talking', 'chatting', or 'complaining' to 'someone' about a problematic situation.

Items 11 and 44 were supposed to be assigned to the factor of avoidance-oriented coping, ${ }^{1}$ but in practice these two items mostly loaded on emotion-oriented coping. In particular, item 44, 'Take time off and get away from the situation' also loaded on the task factor, presenting a similar loading size on both the task- and emotion-oriented coping, but was not shown to load significantly on either factor. This could be explained by the interpretation that the decision to get away from a problematic situation may involve creating space on which to reflect on the problem without the risk of unwelcome confrontation, which in itself does not necessarily constitute avoiding the problem in question. This result is also consistent with the study conducted by McWilliams et al. ${ }^{4}$ which showed that while neither item 11 nor item 44 loaded saliently on any factor, each showed the highest loadings on the emotion factor.

Excepting the three items discussed above, the factorial validity of the results was generally satisfactory and supports Endler and Parker's three-factor model. The congruence coefficients also suggest that the factor structures are very similar in both men and women, and are consistent with the results of Endler and Parker's study, ${ }^{13}$ although the congruence coefficient of factor 3 was not found to be above 0.95 in the present study. Similar conclusions have been drawn by other researchers carrying out their own studies internationally. The insufficient factorial validity shown by previous studies in Korea may have been caused by the inherent psychological instability of adolescents, or perhaps the development of Korean culture in the intervening period has rendered Korean people more susceptible to accurate measurement by the CISS. Either way, as we hope to show in our current study, and contrary to the findings of the previous studies cited, it seems to us that the CISS is indeed applicable to Korean adults in the present day.

One essential element in the localization of any project is accurate translation, and this is no less crucial in the current case. However, this does not appear to have been carried out adequately by those who translated and conducted the original study, the instrument of which we ourselves have used. For 
example, the English phrase 'Go to a party' was translated into Korean as, 'Go to a party. Socialize with friends.' (파티에 간다. 친구들과 만나 논다 - 'Pati-e ganda. Chingudeulgwa manna nonda'). It is clear to us that item 23 would not have shown the highest factor loading in the avoidance-oriented coping category if it had simply read, 'Go to a party', because 'parties' in the Western sense are not part of the Korean cultural milieu. This could be translated into '모임에 간다 - Moim-e ganda' which is a frequently used expression in Korean culture, and its meaning could be re-translated in English as 'attend a gathering/meeting. The matter of accurate localization would have to be taken into account in future studies.

In addition, many studies using the CISS-including validation studies-have been conducted since the early 1990s in Japan, which neighbors Korea and whose culture differs widely from that of North America. Furthermore, the results of such studies appear to indicate the successful adaptation and use of the CISS in Japan while remaining in agreement with the original North American study ${ }^{11,18,30}$ By comparison, research into the applicability of the CISS in a Korean context is still in its infancy, and as such further studies will be required in order to gather sufficient evidence regarding its applicability, or lack thereof. However, despite the detection of certain items displaying equivocal meanings and which require re-assessment, the results of the present study do indeed suggest that the standard version of the CISS may be of use in evaluating the coping strategies of Korean adults as it is. On the other hand, it should be noted that our study also has its limitations, these being chiefly: 1) The small sample size; 2) The absence of testretest reliabilities, and 3) The fact that those who took part were predominantly residents of metropolitan areas. From this, we can infer that we cannot discount the possibility that the broad agreement of our results with the original CISS study may be due in part to the fact that a majority of our participants are more inclined to have been exposed to cultures originating outside Korea, which in turn leaves open the question of whether such agreement would be confirmed should the study be re-conducted in, for example, suburban or rural areas. As such, future studies ought to: use confirmatory factor analysis covering larger sample sizes; to include a wider variety of Korean population samples, and to incorporate improved items.

\section{Acknowledgments}

This study was supported by a Two Year Research Grant of Pusan National University.

\section{REFERENCES}

1. Endler NS, Parker JDA. Assessment of multidimensional coping: task, Emotion, and avoidance strategies. Psychol Assess 1994;6:50-60.

2. Billings AG, Moos RH. The role of coping responses and social re- sources in attenuating the stress of life events. J Behav Med 1981;4:139157.

3. Campbell-Sills L, Cohan SL, Stein MB. Relationship of resilience to personality, coping, and psychiatric symptoms in young adults. Behav Res Ther 2006;44:585-599.

4. McWilliams LA, Cox BJ, Enns MW. Use of the Coping Inventory for Stressful Situations in a clinically depressed sample: factor structure, personality correlates, and prediction of distress. J Clin Psychol 2003; 59:423-437.

5. Lam D, Schuck N, Smith N, Farmer A, Checkley S. Response style, interpersonal difficulties and social functioning in major depressive disorder. J Affect Disorder 2003;75:279-283.

6. Li Z, Zhang J. Coping skills, mental disorders, and suicide among rural youths in China. J Nerv Ment Dis 2012;200:885-890.

7. Billings AG, Moos RH. Coping, stress, and social resources among adults with unipolar depression. J Pers Soc Psychol 1984;46:877-891.

8. Christensen MV, Kessing LV. Clinical use of coping in affective disorder, a critical review of the literature. Clin Pract Epidemiol Ment Health 2005;1:20.

9. Havermans R, Nicolson NA, Devries MW. Daily hassles, uplifts, and time use in individuals with bipolar disorder in remission. J Nerv Ment Dis 2007;195:745-751.

10. MacAulay R, Cohen AS. Affecting coping: does neurocognition predict approach and avoidant coping strategies within schizophrenia spectrum disorders? Psychiatry Res 2013;209:136-141.

11. Nagase Y, Uchiyama M, Kaneita Y, Li L, Kaji T, Takahashi S, et al. Coping strategies and their correlates with depression in the Japanese general population. Psychiatry Res 2009;168:57-66.

12. Vinberg M, Froekjaer VG, Kessing LV. Coping styles in healthy individuals at risk of affective disorder. J Nerv Ment Dis 2010;198:39-44.

13. Endler NS, Parker JD. Multidimensional assessment of coping: a critical-evaluation. J Pers Soc Psychol 1990;58:844-854.

14. Endler NS, Parker JD. Coping Inventory for Stressful Situations (CISS): Manual. Toronto, Canada: Multi-Health Systems; 1990.

15. Calsbeek H, Rijken M, Bekkers MJTM, Henegouwen GPVB, Dekker J. Coping in adolescents and young adults with chronic digestive disorders: impact on school and leisure activities. Psychol Health 2006;21: 447-462.

16. Boysan M. Validity of the Coping Inventory for Stressful Situationsshort form (CISS-21) in a non-clinical Turkish sample. J Psychiatry Neurol Sci 2012;25:101-107.

17. Sakata M, Takagishi Y, Kitamura T. Factor structure of the Japanese version of the Coping Inventory for Stressful Situations: reclassification of coping styles and predictive power for depressive mood. J Psychol Psychother 2013;3:111.

18. Furukawa T, Suzuki-Moor A, Saito Y, Hamanaka T. [Reliability and validity of the Japanese version of the coping inventory for stressful situations (CISS): a contribution to the cross-cultural studies of coping]. Seishin Shinkeigaku Zasshi 1993;95:602-620.

19. Rafnsson FD, Smari J, Windle M, Mears SA, Endler NS. Factor structure and psychometric characteristics of the Icelandic version of the Coping Inventory for Stressful Situations (CISS). Pers Individ Diff 2006; 40:1247-1258.

20. Ramli M, Mohd AF, Khalid Y, Rosnani S. Validation of the Bahasa Malaysia version of the Coping Inventory for Stressful Situation. Malays J Psychiatry 2008;17:7-16.

21. Boysan M. Psychometric properties of the Turkish version of the coping inventory for stressful situations. Arch Neuropsychiatry 2012;49: 196-202.

22. Park YC, Kim KI, Noh S. Validity assessment of the CISS (coping Inventory for stressful situation) in Korean highschool students. J Korean Neuropsychiatr Assoc 2000;39:55-64.

23. Jo HI. Reliability and validity of the Coping Inventory for Stressful Situation (CISS) in African American adolescents. Korean J Counsel Psychotherapy 2000;12:205-214. 
24. Endler NS, Parker JD. Coping Inventory for Stressful Situations (CISS): Manual Multi-Health Systems. Toronto, Canada: Multi-Health Systems; 1999.

25. Johnson DL. A Compendium of Psychosocial Measures: Assessment of People with Serious Mental Illnesses in the Community. New York: Springer; 2010.

26. Jensen AR. The G Factor: the Science of Mental Ability. London: Praeger, Westport, Conn; 1998.

27. Tabachnick BG, Fidell LS. Using Multivariate Statistics. London: Pearson/A\&B, Boston [Mass.]; 2007.
28. Costello AB, Osborne JW. Best practices in exploratory factor analysis: Four recommendations for getting the most from your analysis. Pract Assess Res Eval 2005;10:1-9.

29. Cosway R, Endler NS, Sadler AJ, Deary IJ. The coping inventory for stressful situations: factorial structure and associations with personality traits and psychological health. J Appl Biobehav Res 2000;5:121-143.

30. Uehara T, Sakado K, Sakado M, Sato T, Someya T. Relationship between stress coping and personality in patients with major depressive disorder. Psychother Psychosom 1999;68:26-30 